\title{
Geographic disparities in chronic obstructive pulmonary disease (COPD) hospitalization among Medicare beneficiaries in the United States
}

This article was published in the following Dove Press journal:

International Journal of COPD

7 June 2011

Number of times this article has been viewed

\author{
James B Holt \\ Xingyou Zhang \\ Letitia Presley-Cantrell \\ Janet B Croft \\ National Center for Chronic Disease \\ Prevention and Health Promotion, \\ US Centers for Disease Control and \\ Prevention, Atlanta, GA, USA
}

Correspondence: James B Holt Division of Adult and Community Health, Centers for Disease Control and Prevention, Mailstop K-67, 4770 Buford Highway, NE, Atlanta, GA, USA 3034

$\mathrm{Tel}+\mathrm{I} 7704885510$

Fax +I 7704885965

Email jgh4@cdc.gov
Background: Hospitalizations for persons with chronic obstructive pulmonary disease (COPD) result in significant health care resource use and excess expenditures. Despite well-documented sociodemographic disparities in COPD outcomes, no study has characterized geographic variations in COPD hospitalization across the US.

Methods: Almost 3.8 million COPD hospitalization records were extracted from Medicare claims for 1995-2006, and the total population of eligible Medicare beneficiaries was extracted from the Medicare enrollment records to calculate COPD hospitalization rates by Health Service Area (HSA), $(n=949)$. Spatial cluster analysis and Bayesian hierarchical spatial modeling were used to characterize the geography of COPD hospitalizations.

Results: The overall COPD hospitalization rate was 11.30 per 1,000 beneficiaries for the aggregated period 1995-2006. HSA-level COPD hospitalization rates had a median of 11.7 and a range of 3.0 (Cache, UT) to 76.3 (Pike, KY). Excessive hospitalization risk was concentrated in Appalachia, the southern Great Lakes, the Mississippi Delta, the Deep South, and west Texas. In the Bayesian spatial mixture model, $73 \%$ of variability of COPD hospitalization relative risk was attributed to unidentified regional social and physical environments shared by HSAs rather than to unique local HSA factors (27\%).

Conclusion: We discovered distinct geographic patterns in COPD hospitalization rates and risks attributed to both regionally-shared environmental risk factors and HSA-unique environmental contexts. The correlates of these geographic patterns remain to be determined. Geographic comparisons of COPD hospitalization risk provide insights for better public health practice, policies, and programs for COPD prevention.

Keywords: COPD prevention, environmental risk factors, public health, population

\section{Introduction}

Chronic obstructive pulmonary disease (COPD) is a heterogeneous group of slowly progressive diseases characterized by airflow limitation and gradual loss of lung function that is not fully reversible. ${ }^{1}$ COPD represents $5 \%$ of total deaths and is the fourth leading cause of death in the US. The absolute number of deaths from COPD increased $8 \%$ from 116,494 in 2000 to 126,005 in $2005 .^{2}$ During 1980-2000, the overall death rate for COPD increased $67 \% .{ }^{3}$ The COPD average attributable excess health care expenditures are nearly US\$6,300 per Medicare patient. ${ }^{4}$ The annual number of inpatient days for COPD (8.18 days) is only second to chronic kidney disease (9.51 days). ${ }^{5}$ COPD is highly prevalent and associated with comorbid disease and physical dysfunction. ${ }^{6}$

Significant sociodemographic disparities in COPD outcomes have been reported for the US. Only a few studies explored their geographic variations..$^{7-10}$ A broader 
view of the causes of population health disparities including race, ethnicity, socioeconomic status, and geography is necessary for better solutions to complex population health problems. ${ }^{11}$ Understanding geographic variations in COPD hospitalization could help public health policy makers more clearly identify target areas with great needs and facilitate the formulation of better solutions to improve COPD prevention and treatment. No previous study has characterized the geographic variations in COPD hospitalization across the US. This study examined geographic disparities in COPD hospitalization of Medicare beneficiaries in the US, at the state and Health Service Area (HSA) level. The geographic variations in regionalized and local environmental factors that contributed to excessive HSA hospitalization risk were then assessed.

\section{Methodology}

\section{Data}

COPD hospitalization data were extracted from $100 \%$ of the Part A Medicare claims for 1995-2006. The number of Medicare hospitalizations for which COPD was the primary discharge diagnosis using International Classification of Diseases, Ninth Revision (ICD-9) codes 490 (bronchitis not specified as acute or chronic), 491 (chronic bronchitis), 492 (emphysema), and 496 (chronic airway obstruction, not elsewhere classified) were obtained. Medicare enrollment records were used as the denominator file to calculate the total population of eligible Medicare beneficiaries (limited to those aged 65 and older in fee-for-service Medicare) for each HSA $(n=949)$.

The outcome measure is the COPD Medicare beneficiary hospitalization rate over a 12-year study period (1995-2006) by HSA (we chose to use the National Cancer Institute's modified HSAs to ensure that all HSAs were fully contained within discrete states and that no state boundary crossings occurred, as is the case with the original HSAs defined by the National Center for Health Statistics). HSAs were selected as the geographic units of analysis for three main reasons. First, smaller geographic units (eg, counties) would have small numbers of Medicare hospitalizations and enrollee populations, which yield statistically unstable estimates of COPD hospitalization rates. Second, HSAs were designated for effective planning and development of health services. Similar healthcare resource delivery patterns within a HSA may be relevant to its residents' COPD hospitalization healthcare needs. Finally, state level aggregations would smooth out significant sub-state variations of COPD hospitalization. The 12-year temporal aggregation resulted in a minimum of 8 COPD hospitalizations and a Medicare population of at least 1,235 for all 949 HSAs, which provides a base for reliable estimation of HSA-level COPD hospitalization rates and risk.

\section{Statistical analysis and modeling}

The preliminary descriptive analysis included crude rates and maps. Statistical analysis began with spatial cluster analysis to explore spatial continuities and discontinuities of COPD hospitalization rates. Spatial continuities (or discontinuities) indicate that COPD hospitalization rates and risk exhibit smooth (or disruptive) variation between adjacent geographic areas. Namely the rate or risk surface of COPD hospitalization has a smooth (or discordant) spatial pattern among neighboring areas. These smooth or discordant patterns could be attributable to a number of as yet undefined factors. A Bayesian hierarchical spatial modeling approach was applied to characterize the geography of COPD hospitalization risk. The goal was to identify which HSAs or regions have significantly higher or lower COPD hospitalization risks compared to the average HSA level risk, and whether HSA local or regional characteristics, or both, may contribute to HSAs' excessive or reduced COPD hospitalization risks. Regional characteristics are those environmental/ contextual conditions that extend over a large area, perhaps encompassing several states (eg, Appalachia) - these may represent widespread spatial processes, such as regionalized poverty. HSA local characteristics represent environmental/ contextual conditions that occur in smaller areas (eg, one or a few contiguous counties) - these may represent place-specific conditions, such as localized occupational exposures.

State and HSA level COPD hospitalization rate maps (Figures 1 and 2) showed large geographic variations and strong spatial continuities and some discontinuities over the US. Using STIS software (TerraSeer, Inc), the Moran I index ${ }^{12}$ was computed to assess the overall spatial autocorrelation of HSA COPD hospitalization rates across the US, while local indicators of spatial association ${ }^{13}$ were computed to examine their local spatial dependence and discontinuities. Local spatial cluster analysis classified HSAs into the following five categories: 1) spatial clusters of HSAs with significantly higher rates than surrounding HSAs; 2) spatial clusters of HSAs with significantly lower rates than the surrounding HSAs; 3) spatial outliers of HSAs with significantly higher rates than surrounding HSAs; 4) spatial outliers of HSAs with significantly lower rates than surrounding HSAs; and 5) HSAs that have no significant spatial autocorrelation with surrounding HSAs for COPD hospitalization rates. 


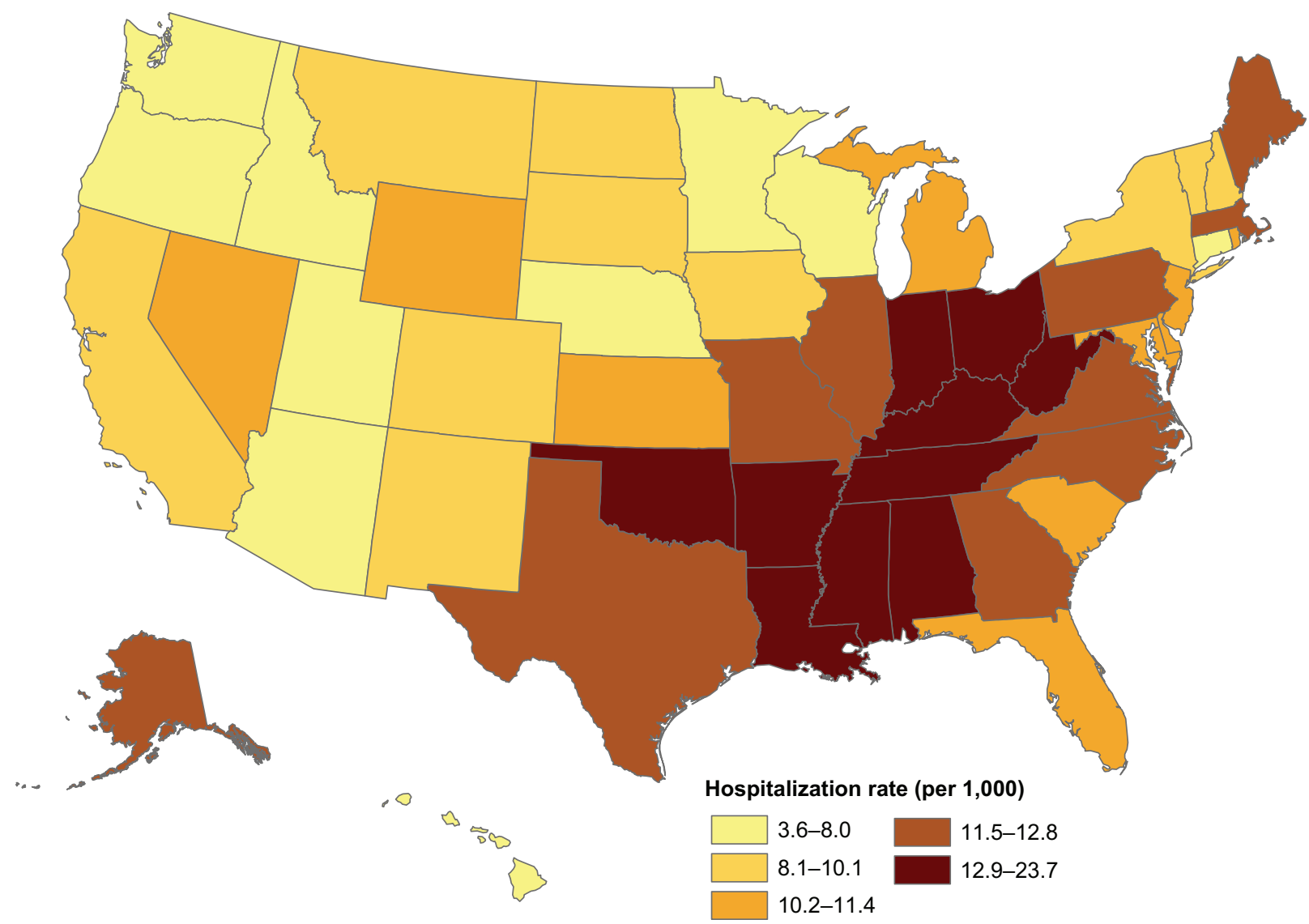

Figure I COPD hospitalization rate (per I,000 enrollees) among Medicare beneficiaries by state (1995-2006).

Bayesian hierarchical spatial modeling is commonly used in disease mapping and small area risk assessment. ${ }^{14}$ A Bayesian spatial mixture mode ${ }^{15}$ was constructed, which extends the classical spatial convolution mode ${ }^{16}$ by introducing a spatial mixture parameter to balance the spatially smooth and disruptive effects on COPD outcomes. The spatial mixture model was implemented via Markov Chain Monte Carlo methods in WinBUGS. ${ }^{17}$

\section{Results}

\section{Descriptive analysis}

There was a total of 3,786,908 Medicare beneficiaries hospitalized with COPD in the US between 1995 and 2006, (mean $=315,576 /$ year). The 1995-2006 average COPD hospitalization rate was 11.30 per 1,000 Medicare beneficiaries (Table 1). The annual rates exhibited no significant temporal trend; the rate increased from 10.46 per 1,000 in 1995 to 13.38 per 1,000 in 1999 and then decreased to 10.21 per 1,000 in 2006. However, there were significant differences among states and HSAs. At state level, Utah (3.6 per 1,000) and Hawaii $(4.5$ per 1,000) had the lowest rates, while West Virginia $(23.7$ per 1,000$)$ and Kentucky $(20.8$ per 1,000$)$ had the highest rates (Figure 1). Large portions of the Mississippi Delta, the Deep South, and Appalachia formed a large geographic cluster with significantly higher rates, while northern and western states had lower rates.

COPD hospitalization rates had larger geographic variations in HSAs than in states, with a median of 11.7 per 1,000 and a range of 3.0 per 1,000 (Cache, UT) to 76.3 per 1,000 (Pike, KY) (Figure 2). Wide contrasts between the state rate and a 2 to 3 times higher HSA rate occurred in West Virginia, North Dakota, Kentucky, Oklahoma, Arkansas, and Virginia. In contrast, some HSAs in Minnesota, Idaho, Iowa, Nebraska, Mississippi, Kansas, and Montana had rates that were less than half of the rates of their respective states. The five states with the largest percentage point range in intrastate HSA-specific rates were Kentucky (64.49), West Virginia (55.69), Virginia (51.87), Arkansas (40.30), and Oklahoma (35.74). The five states with the smallest percentage point range in HSA-specific rates were Rhode Island (1.28), Delaware (1.68), New Jersey (3.23), Nevada (3.80), and Connecticut (4.42). With few exceptions, states with the highest and lowest ranges of intrastate rates were highly clustered geographically. 


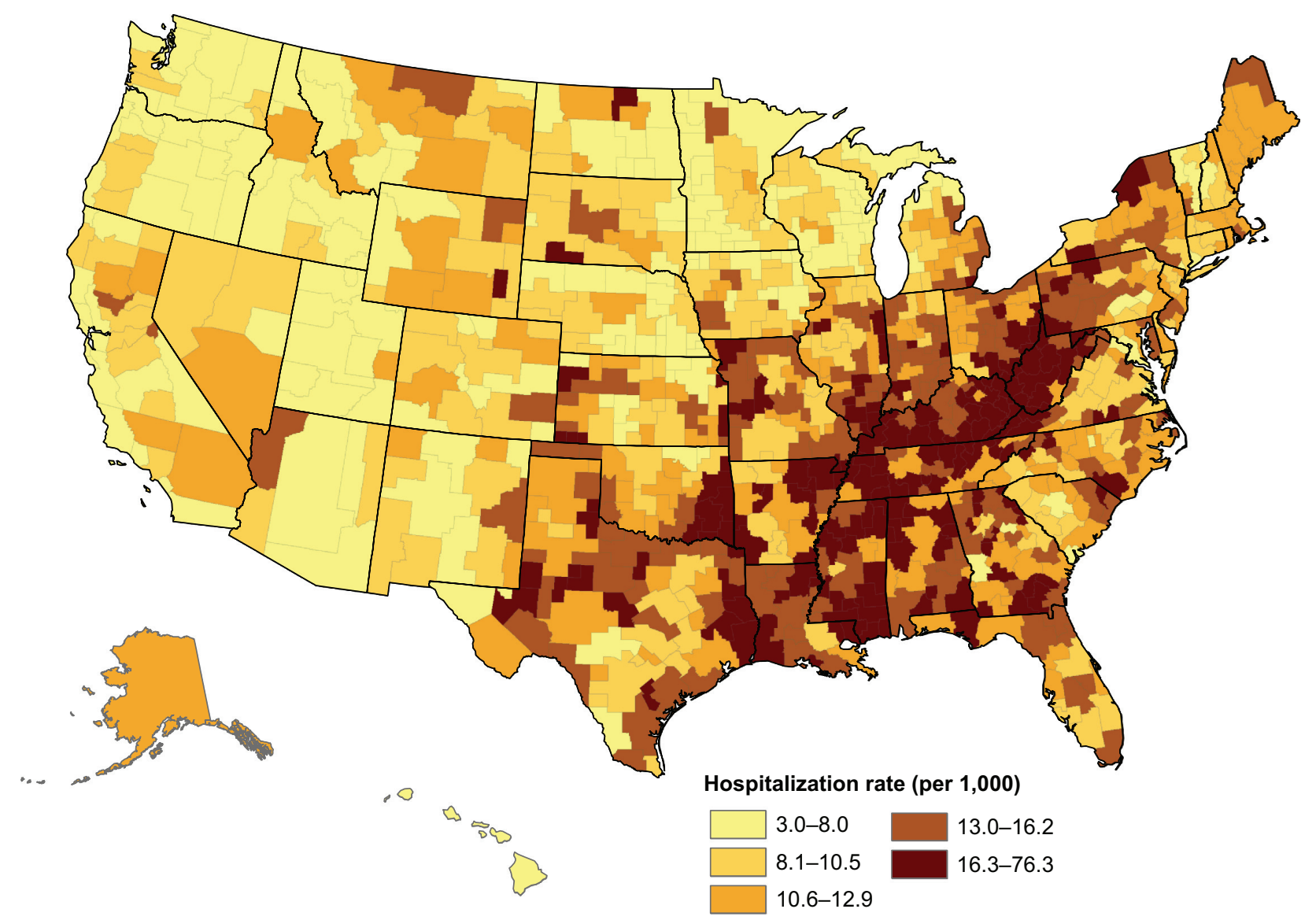

Figure 2 COPD hospitalization rate (per I,000 enrollees) among Medicare beneficiaries by HSA (1995-2006).

\section{Spatial analysis}

Moran's I for HSA COPD hospitalization rates (1995-2006) had a value of $0.59(P<0.0001)$, confirming a significant positive spatial autocorrelation at the HSA level for COPD hospitalization rates across the US. Local spatial cluster analysis identified 35 HSAs within spatial clusters of

Table I Medicare beneficiary COPD hospitalizations rates (per I,000 enrollees) by year

\begin{tabular}{llll}
\hline Year & $\begin{array}{l}\text { Medicare } \\
\text { population }\end{array}$ & $\begin{array}{l}\text { COPD } \\
\text { hospitalization }\end{array}$ & $\begin{array}{l}\text { Rate } \\
\text { (per 1000) }\end{array}$ \\
\hline 1995 & $28,584,063$ & 299,112 & 10.46 \\
1996 & $28,052,524$ & 304,218 & 10.84 \\
1997 & $27,289,171$ & 320,298 & 11.74 \\
1998 & $26,525,251$ & 321,774 & 12.13 \\
1999 & $26,198,615$ & 350,659 & 13.38 \\
2000 & $26,564,681$ & 318,740 & 12.00 \\
2001 & $27,378,646$ & 322,811 & 11.79 \\
2002 & $28,191,343$ & 326,379 & 11.58 \\
2003 & $28,704,729$ & 317,275 & 11.05 \\
2004 & $29,059,259$ & 290,993 & 10.01 \\
2005 & $29,160,779$ & 313,635 & 10.76 \\
2006 & $29,483,742$ & 301,014 & 10.21 \\
Overall & $335,192,803$ & $3,786,908$ & 11.30 \\
\hline
\end{tabular}

high rates, 163 HSAs within spatial clusters of low rates, 2 high-rate spatial outliers (Beltrami-Clearwater HSA in Minnesota and Alpine HSA in California), and 1 low-rate spatial outlier (Greenbrier-Monroe HSA in West Virginia). Spatial clusters of high rates were concentrated in central Appalachia and northeast Arkansas. Spatial clusters of low rates were located in broad areas of the upper Midwest and central Great Plains, the Rocky Mountains and Great Basin, the Pacific Northwest, and California.

The Bayesian spatial mixture model showed that nearly $73 \%$ variability of the COPD hospitalization relative risk was attributed to the spatially structured effects. Thus, the HSAs' regionalized social and physical environments have a greater influence on COPD hospitalization than the HSAs' local environmental risk factors. This finding is consistent with the strong positive spatial autocorrelation from spatial cluster analysis. These social and physical environments have not yet been identified in the current report.

\section{Geography of COPD hospitalization risk}

The geography of excessive or reduced risk for COPD hospitalization was estimated using the posterior probability 
from the Bayesian spatial mixture model. The relative risks attributable to undefined regional and local environmental influences were mapped in Figures 3 and 4 respectively. A large, contiguous area of 377 HSAs with significant spatially structured excessive risk (probability exceeding 0.8) was concentrated in Appalachia, the southern Great Lakes states, the Mississippi Delta, the Deep South, and west Texas. By contrast, 38 HSAs with significant unstructured local excessive risk (probability exceeding 0.8 ) were sparsely spread over the northern Great Plains and along the East Coast. Reduced hospitalization risks due to regional environmental factors were observed in 355 HSAs mainly in the western US, while 59 HSAs in the eastern and south central US had reduced hospitalization risks that were potentially associated with local unique environment factors.

\section{Discussion}

Medicare claims data were used to characterize substantial geographic variations in COPD hospitalization risk among states (Figure 1) and HSAs (Figure 2) for individuals aged 65 and older in fee-for-service Medicare. The HSA-level analysis highlights high hospitalization burdens within states whose overall rates may not be high relative to other states. This geographic analysis facilitates identification of target areas of high need, leading to more efficient and effective use of resources. There were distinct geographic patterns in COPD hospitalization risks attributed to regionally-shared environmental risk factors (Figure 3) and HSA-unique environmental contexts (Figure 4). These undefined environmental risk factors for HSA COPD hospitalization are strongly spatially correlated among those neighboring HSAs, while a few HSAs could attribute their COPD hospitalization risk to their unique local environmental context. These findings suggest that two spatial processes may be operating with respect to environmental influences on COPD hospitalization; first, broad regionalized contextual effects (eg, socioeconomic factors and high regionalized population smoking rates) may be exacerbating COPD hospitalizations. Second, localized environmental factors, such as occupational exposures, may be influencing COPD hospitalizations. Further studies are needed to understand these specific regional and local environmental factors contributing to excess or reduced COPD hospitalization risk.

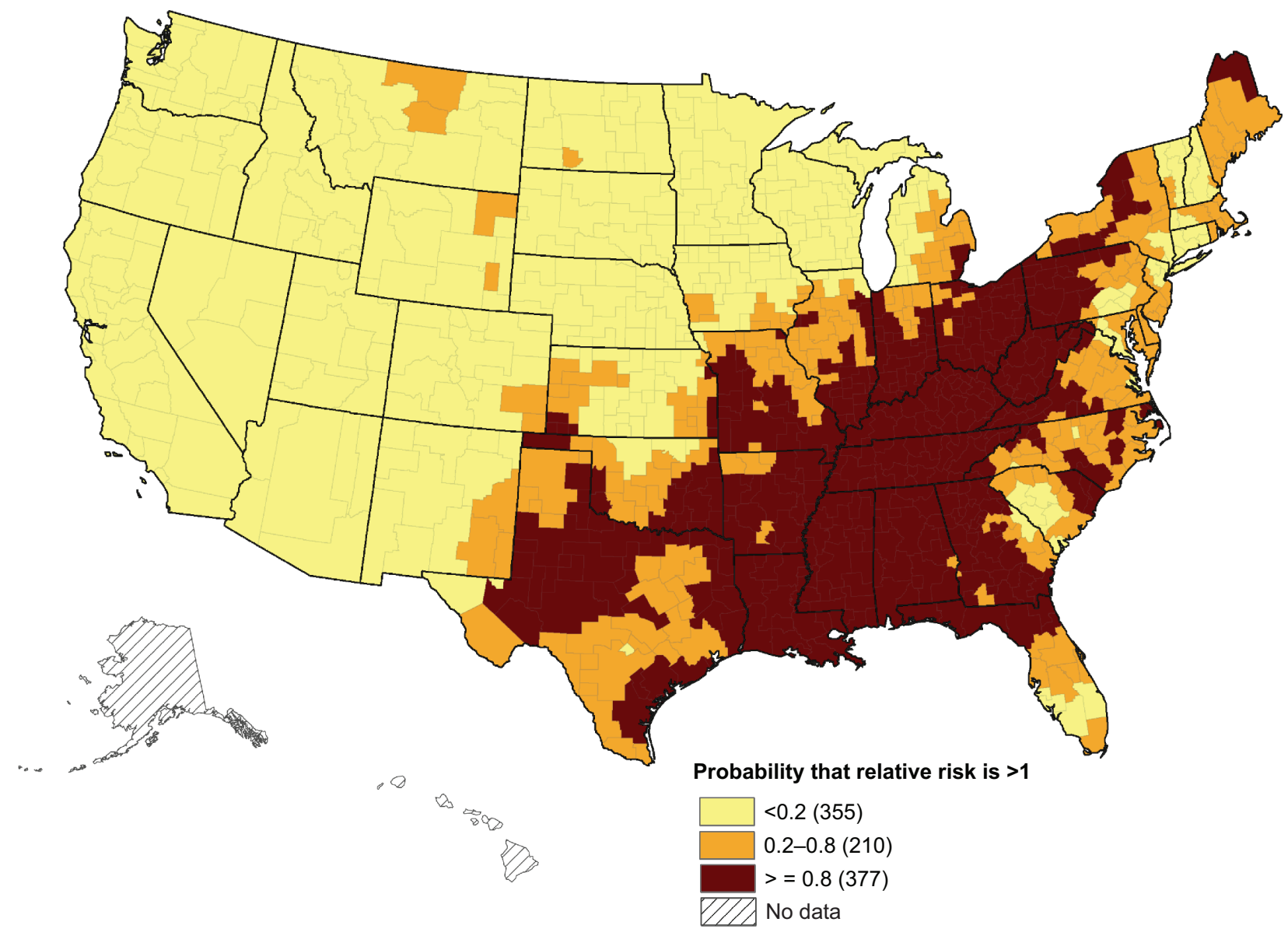

Figure 3 COPD hospitalization relative risk exceeding I, attributable to regional environmental factors. 


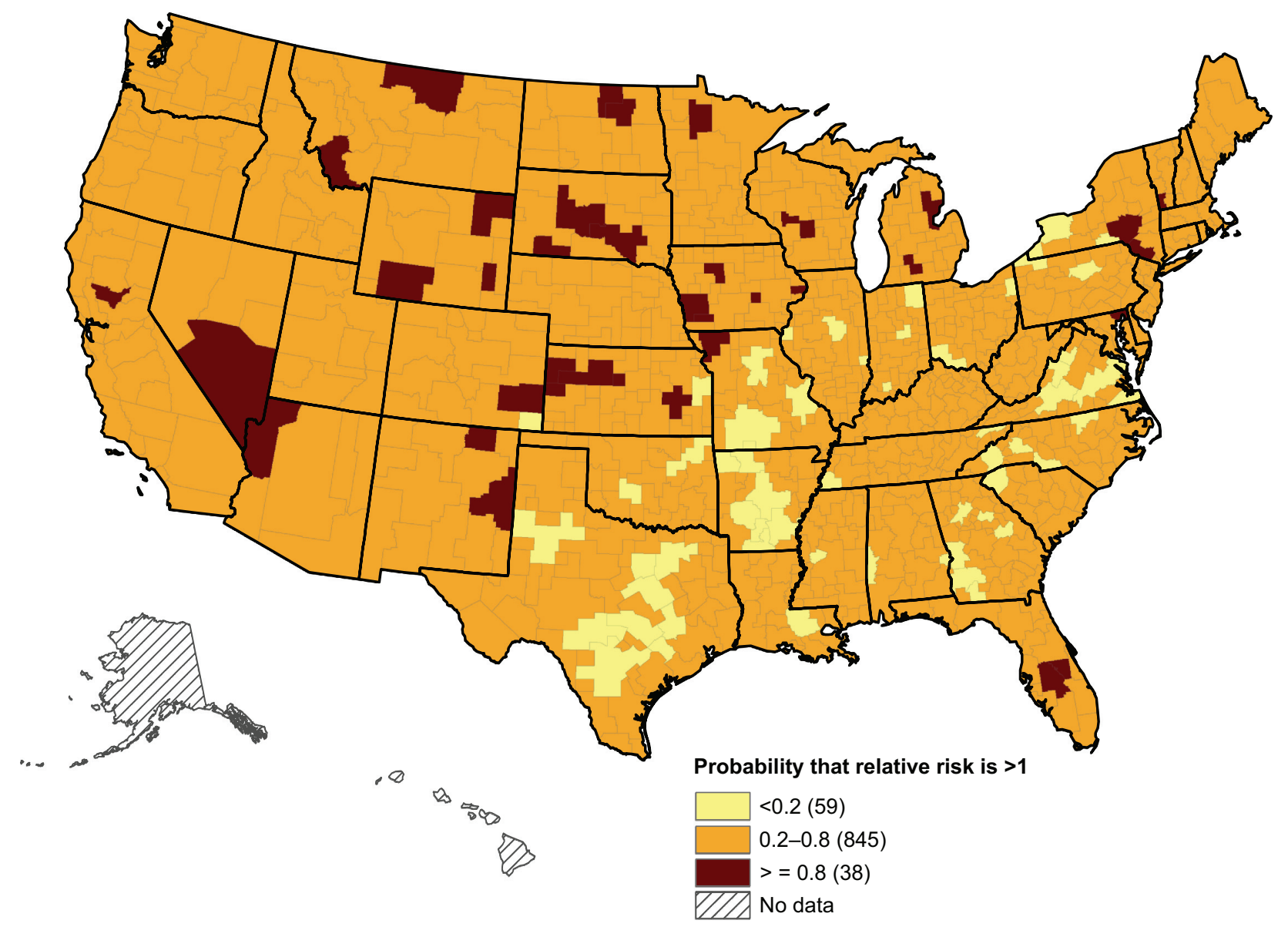

Figure 4 COPD hospitalization relative risk exceeding I, attributable to local environment factors.

This analysis yields valuable insights into the potential ecological factors that could explain geographic variations in COPD. In turn, this could help to generate hypotheses for further research. For example, the large area of increased hospitalization risk due to regionalized factors (Figure 3) might suggest the inclusion of a socioeconomic status variable such as poverty, as an area-level covariate in further models. In contrast, areas with increased hospitalization risk due to localized factors (for example, many of the HSAs in the western US, shown in Figure 4) might suggest the inclusion of area-level covariates such as the percentage of the population employed in mining or agriculture, in which occupational exposures have been hypothesized to exacerbate COPD, thus leading to higher hospitalization rates.

The drivers of these significant geographic disparities is far from simple, because COPD hospitalization does not only reflect medical care, because also the severity of the COPD and other co-existing chronic conditions. ${ }^{5}$ There is evidence to suggest that the geographic disparities in COPD hospitalization could be explained mainly by classical risk factors such as smoking, a well-documented individual risk factor for COPD mortality and morbidity. ${ }^{18}$ However there exists a significant temporal and spatial mismatch between population smoking and COPD hospitalization rates. If smoking is a dominant contributor of COPD hospitalization, one would expect to observe a significant decreasing COPD hospitalization rate in the US. Also, from the geographic perspective, western states (eg, North Dakota, Montana, and Wyoming) have relative high smoking rates ${ }^{19}$ but low COPD hospitalization rates. Yet, high smoking prevalence is consistent with high COPD hospitalization rates in Appalachian areas and neighboring regions. This geographic match and mismatch between population smoking prevalence and COPD hospitalization rates suggests that environment risk factors may have geographically differential relationships with COPD hospitalization risk. Population smoking could be a major contributor of COPD hospitalization in some areas while other environmental risk factors, such as air pollution (indoor and outside) $)^{20-22}$ and occupational exposures (mining, farming, and construction $)^{23-27}$ or exposure to biomass heating fuel, ${ }^{20,28,29}$ could play a dominant role in other areas. 
Brown describes significant state-level geographic variations in COPD mortality. ${ }^{7}$ A state-level comparison between COPD hospitalization and mortality rates shows significant geographic matches and mismatches across the US. This again indicates that these two COPD outcomes share some commonalities in their risk factors but also have differences in their potential sets of risk factors. A further Bayesian spatial mixture model with both COPD outcomes (hospitalization and mortality) at the same time may disentangle the potential factors behind their geographic patterns.

There are a few limitations of this study. First, the study population includes only those individuals age 65 and older who are enrolled in fee-for-service Medicare. It does not include younger individuals or those who are receiving care funded through private savings or other insurance. Having data on these populations would help to better understand COPD hospitalization patterns and factors that could potentially explain geographic variations.

Second, this is an ecological study with potential for ecological fallacy or bias. Observed geographic variations in COPD hospitalization might be a consequence of the different demographic composition in Medicare enrollees. For example, COPD is the third leading cause of mortality for the age group 65-84 and fourth leading cause for the age group of 85 and above..$^{30}$ Geographic variations in COPD hospitalization should be affected by regional elderly population compositions. However, because Medicare Claims data does not provide accurate measures of individual risk factors, such as smoking and occupation exposure, ecological studies provide a viable framework to evaluate the environmental exposures on COPD hospitalization.

Third, geographic differences in COPD hospitalization may change over time. This study was based on aggregated 12-year Medicare claims data; therefore it was not possible to determine whether the spatial pattern of COPD hospitalization is stable over the study period. Further research is needed to examine the spatiotemporal changes in COPD hospitalization to confirm the implicit assumption of its stable spatial configuration.

COPD is a chronic disease that can be prevented and treated. COPD hospitalization is a costly and sometimes life-threatening event associated with a variety of environmental risk factors, including access to health care. ${ }^{1,31-34}$ The geographic variation of COPD hospitalization highlights the potential and possibility of reducing its geographic disparities. From population health policy, the observed geographic gaps in COPD hospitalization risk could be the consequence of different regional environmental exposures, but it may be more a result of different COPD community prevention efforts. The variation among states in COPD hospitalization suggests reducing COPD hospitalization burden may be possible on a national scale; although this study did not investigate the exact environmental exposures or community healthcare practices that cause these differences. The differences in COPD hospitalization rates among states and among HSAs further pinpoints the potential local areas which could benefit from enhanced public health efforts to prevent and reduce COPD hospitalization, even among those states with overall lower COPD hospitalization rates. Geographic comparison of lower and higher regional COPD hospitalization risk could also provide insights for better public health practice for COPD prevention. This study focused on the geography of COPD hospitalization burden and risk in order to facilitate public health policy delivery for COPD prevention and to improve public health practice strategies to reduce COPD hospitalization. Future studies could examine the impact of programs such as the National Heart, Lung, and Blood Institute's (NHLBI) "Learn More Breathe Better" campaign. The NHLBI campaign is focused in the US and seeks to develop better public health policies and strategies in partnership with primary care providers, public health professionals, and persons with or at risk for COPD to reduce the morbidity and mortality burden associated with COPD by increasing awareness and promoting effective prevention and treatment strategies.

\section{Disclosure}

This work was conducted at the Centers for Disease Control and Prevention. The findings and conclusions in this report are those of the authors and do not necessarily represent the official position of the Centers for Disease Control and Prevention.

\section{References}

1. Mannino DM. Chronic obstructive pulmonary disease: definition and epidemiology. Respir Care. 2003;48(12):1185-1191; discussion 1191-1193.

2. Centers for Disease Control and Prevention. Deaths from chronic obstructive pulmonary disease - United States, 2000-2005. MMWR. 2008:57(45).

3. Mannino D, Hom D, Akinbami L, Ford E, Redd S. Chronic obstructive pulmonary disease surveillance - United States, 1971-2000. WWMR 2002;51(SS-6):1-16.

4. Menzin J, Boulanger L, Marton J, et al. The economic burden of chronic obstructive pulmonary disease (COPD) in a US Medicare population. Respir Med. 2008;102(9):1248-1256

5. Schneider KM, O’Donnell BE, Dean D. Prevalence of multiple chronic conditions in the United States' Medicare population. Health Qual Life Outcomes. 2009;7:82. 
6. Methvin JN, Mannino DM, Casey BR. COPD Prevalence in Southeastern Kentucky: The Burden of Lung Disease Study. Chest. 2009; 135(1):102-107.

7. Brown D, Croft J, Greenlund K, Giles W. Deaths from chronic obstructive pulmonary disease - United States, 2000-2005. MMWR. 2008; 57:45.

8. Joo MJ, Lee TA, Weiss KB. Geographic variation in chronic obstructive pulmonary disease exacerbation rates. J Gen Intern Med. 2007;22: $1560-1565$.

9. Lipton R, Banerjee R. The geography of chronic obstructive pulmonary disease in California, 1993 and 1999: using a novel method to transform zip code level data to a uniform grid structure. Am J Epidemiol. 2006;163(11):S28-S28.

10. Nandram B, Sedransk J, Pickle LW. Bayesian analysis and mapping of mortality rates for chronic obstructive pulmonary disease. J Am Statist Assoc. 2000;95(452):1110-1118.

11. Frist WH. Overcoming disparities in US health care - a broad view of the causes of health disparities can lead to better, more appropriate solutions. Health Affairs. 2005;24(2):445-451.

12. Moran P. Notes on continuous stochastic phenomena. Biometrika. 1950;37:17-33.

13. Anselin L. Local indicators of spatial association-LISA. Geogr Anal. 1995;27(2):93-115.

14. Lawson AB. Bayesian disease mapping. First ed. New York: CRC Press; 2008.

15. Congdon P. Mixtures of spatial and unstructured effects for spatially discontinuous health outcomes. Comput Stat Data Anal. 2007;51: 3197-3212.

16. Besag J, York J, Mollie A. Bayesian image-restoration, with 2 applications in spatial statistics. Ann Inst Stat Math. 1991;43(1):1-20.

17. WinBUGS Version 1.4 [computer program]. 2003.

18. Viegi G, Scognamiglio A, Baldacci S, Pistelli F, Carrozzi L. Epidemiology of chronic obstructive pulmonary disease (COPD). Respir Int Rev Thorac Dis. 2001;68(1):4-19.

19. Centers for Disease Control and Prevention. State-specific prevalence and trends in adult cigarette smoking - United States, 1998-2007. MMWR. 2009;58(9):221-226.

20. Zhang JJ, Smith KR. Household air pollution from coal and biomass fuels in China: measurements, health impacts, and interventions. Environ Health Perspect. 2007;115(6):848-855.
21. Donaldson GC. C-reactive protein: does it predict mortality? Am J Respir Crit Care Med. 2007;175(3):209-210.

22. Abbey DE, Hwang BL, Burchette RJ, Vancuren T, Mills PK. Estimated long-term ambient concentrations of PM10 and development of respiratory symptoms in a nonsmoking population. Arch Environ Health. 1995;50(2):139-152.

23. Bang KM, Syamlal G, Mazurek JM. Prevalence of chronic obstructive pulmonary disease in the US working population: an analysis of data from the 1997-2004 National Health Interview Survey. COPD. 2009; 6(5):380-387.

24. Greskevitch M, Kullman G, Bang KM, Mazurek JM. Respiratory disease in agricultural workers: mortality and morbidity statistics. J Agromed. 2007;12(3):5-10.

25. Hnizdo E, Sullivan PA, Bang KM, Wagner G. Association between chronic obstructive pulmonary disease and employment by industry and occupation in the US population: a study of data from the Third National Health and Nutrition Examination Survey. Am J Epidemiol. 2002;156(8):738-746.

26. Hnizdo E, Sullivan PA, Bang KM, Wagner G. Airflow obstruction attributable to work in industry and occupation among US race/ethnic groups: a study of NHANES III data. Am J Indust Med. 2004;46(2):126-135.

27. Hnizdo E, Vallyathan V. Chronic obstructive pulmonary disease due to occupational exposure to silica dust: a review of epidemiological and pathological evidence. Occup Environ Med. 2003;60(4):237-243.

28. Akhtar T, Ullah Z, Khan MH, Nazli R. Chronic bronchitis in women using solid biomass fuel in rural Peshawar, Pakistan. Chest. 2007;132(5): 1472-1475.

29. Kurmi OP, Semple S, Simkhada P, Smith WC, Ayres JG. COPD and chronic bronchitis risk of indoor air pollution from solid fuel: a systematic review and meta-analysis. Thorax. 2010;65(3):221-228.

30. Heron M, Tejada-Vera B. National vital statistics reports. Hyattsville, MD: National Center for Health Statistics; 2009.

31. Leigh JP, Romano PS, Schenker MB, Kreiss K. Costs of occupational COPD and asthma. Chest. 2002;121(1):264-272.

32. Mannino DM. COPD - epidemiology, prevalence, morbidity and mortality, and disease heterogeneity. Chest. 2002;121(5):121s-126s.

33. Mannino DM. Epidemiology and global impact of chronic obstructive pulmonary disease. Sem Respir Crit Care Med. 2005;26(2):204-210.

34. Strassels SA, Smith DH, Sullivan SD, Mahajan PS. The costs of treating COPD in the United States. Chest. 2001;119(2):344-352.
International Journal of COPD

\section{Publish your work in this journal}

The International Journal of COPD is an international, peer-reviewed journal of therapeutics and pharmacology focusing on concise rapid reporting of clinical studies and reviews in COPD. Special focus is given to the pathophysiological processes underlying the disease, intervention programs, patient focused education, and self management protocols.

\section{Dovepress}

This journal is indexed on PubMed Central, MedLine and CAS. The manuscript management system is completely online and includes a very quick and fair peer-review system, which is all easy to use. Visit $\mathrm{http}: / /$ www.dovepress.com/testimonials.php to read real quotes from published authors. 